\title{
Medic al intensive care for lung cancer patients: better than we thought?
}

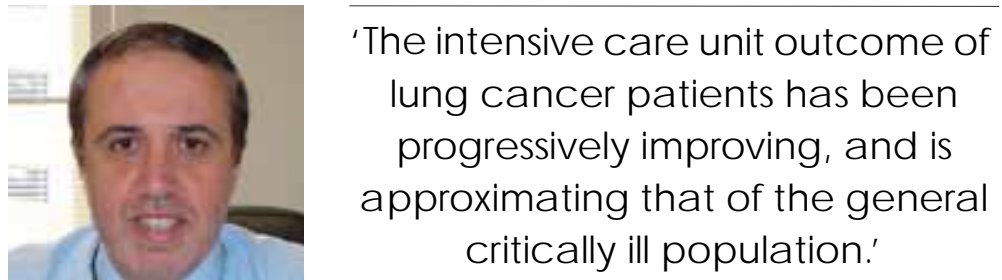

Lung cancer is the third most common malignancy, but remains the leading cause of cancerrelated death in males and females throughout the world [1]. The management of lung cancer in the last two decades has been marked by more aggressive surgical procedures, and an intensive combination of chemotherapy and radiation. This has resulted in an increasing number of patients being admitted to the intensive care unit (ICU) for the management of critical illness related to advanced underlying malignancy, complications of therapy, or comorbid illnesses. Several studies have shown that the most common indications for ICU care in this patient population are related to acute respiratory failure secondary to underlying malignancy. These include massive malignant pleural effusions, airway infiltration by tumor leading to the obstruction of main airways, massive hemoptysis or treatment-related pneumonitis. Other pulmonary problems may also lead to admission to the ICU, such as pneumonia, acute exacerbation of chronic obstructive pulmonary disease or pulmonary embolism. Given their age and comorbidities, lung cancer patients may need ICU care as a result of severe sepsis, cardiac disease (such as myocardial infarction or congestive heart failure) or neurological problems (such as cerebrovascular accident, intracerebral bleeding or change in mental status) [2-5].

Lung cancer is the third most common solid tumor in critically ill patients, and accounts for $16 \%$ of all malignancies in cancer admissions to the ICU [6,7]. Earlier studies showed that the ICU mortality rate of lung cancer was $70-85 \%$, and the hospital mortality rate reaches $90 \%[8,9]$. Furthermore, the economic cost of this care is significant. In a study of elderly lung cancer patients (aged $\geq 66$ years), the I CU use in the last 6 months of life increased from 17.5 to $24.7 \%$ from 1993 to 2002. The total healthcare cost was
US\$40,929 for ICU patients compared with U $\$ 27,160$ for non-ICU patients $(p<0.001$ ) [5]. H owever, recent studies have shown that the outcome of these patients has been steadily improving. In a study from Europe and South America of 143 lung cancer patients admitted to the ICU, including 100 mechanically ventilated patients, the hospital mortality rate was $59 \%$ [3]. Another more recent study from the USA, by Adam and Soubani, studied 139 lung cancer patients admitted to the ICU, excluding those admitted for post-lung-resection monitoring. The ICU mortality rate was $22 \%$ and the hospital mortality rate was $40 \%$, which is similar to those of the general patient population admitted to the ICU [2]. It is difficult to make a firm statement regarding the reasons for the improved outcome of lung cancer patients, although factors that have been shown to improve ICU outcome in other patient populations probably apply to these patients as well. These factors include the increased use of noninvasive positive-pressure ventilation for patients with acute respiratory failure, lung protective strategies for mechanically ventilated patients and improved approaches to the management of severe sepsis. In the case of noninvasive positive pressure ventilation, a few studies report that the early use of this strategy has resulted in improved gas exchange, decreased dyspnea, less mechanical ventilation, and lower overall mortality rates $[10,11]$. In addition, the increased knowledge and experience that develops in ICUs that routinely deal with patients with malignancy regarding their unique complications may play a role. Furthermore, the multidisciplinary approach that symbolizes modern ICU care and includes intensivists, oncologists and other specialists probably contributes to the better management of these patients.

D espite the improved ICU and hospital outcome of lung cancer patients reported in the recent literature, not all patients benefit from this aggressive care. For many of the patients with lung cancer, ICU care is futile and will not prolong their lives. Such therapy may be associated with a huge emotional and physical toll on these patients and their families. In addition, such care will consumescarce resources and is very expensive. 
It would be useful to be able to predict, prior to admission to the ICU, whether the patient is going to benefit from this aggressive and expensive therapy. The retrospective studies on this patient population looked at different variables. The type of lung cancer and the stage have not been shown to be predictors of ICU outcome [9]. Furthermore, age does not predict the ICU outcome in this patient population and should not be used as a major factor in determining the futility of ICU care; rather, the performance status of the patient appears to be a more significant factor. A recent study of 69 cancer cases, including lung cancer, admitted to the ICU reported that only performance status (3-4) was a predictor of short-term negative outcome of ICU care [12]. Acuity scoring systems upon admission to the ICU, such as Acute Physiology and Chronic $\mathrm{H}$ ealth Evaluation (APACHE) II and III or Simplified Acute Physiology Scores III, have also been extensively studied. Most of the studies showed that these systems underestimate mortality and are not a useful predictor of outcome in patients with malignancy, including lung cancer [13]. The need for mechanical ventilation is another variable that has been studied and proven to bean important predictor of poor ICU and hospital outcome in lung cancer patients $[9,10,14]$. The difference in survival is significant between critically ill lung cancer patients who are mechanically ventilated and those who are not (hospital mortality: 53 vs $27 \%$, respectively) [2]. H owever, even those lung cancer patients who required mechanical ventilation have been showing consistent improvement in outcome, with ICU mortality of 38\% and hospital mortality of $53 \%$ [2]. Another predictor that has been reported in more than one study is the presence of multiorgan failure $[3,9,13]$. The presence of two or more organs with dysfunctions was significantly associated with poor ICU outcome (OR: 40.8; 95\% Cl: 2.1-328.3; $p<0.0001$ ) [2]. This trend was seen with all types of organsystem failure. 0 ther variables that have been described in some studies, al beit not consistently, include increased serum lactate level on admission to the ICU, the need for vasopressor therapy, infiltration of the airway by tumor and the severity of comorbid illnesses [2,3].

It is clear from the available studies that there are no absolute predictors of ICU outcome of lung cancer patients. It is also certain that the outcome is more dependent on the severity of the acute illness and the number of organ failures than the characteristics of the underlying malignancy.
Given the difficulty in determining the futility of ICU care for patients with lung cancer upon initial evaluation of their critical illness, it is reasonable to offer ICU care to all critically ill lung cancer patients except those who have advanced malignancy unresponsive to therapy, or have made a decision against aggressive therapy. Treatment in these patients admitted to the ICU should include appropriate diagnostic studies, ventilatory support (either by noninvasive positive-pressure ventilation or mechanical ventilation), hemodynamic support and, when necessary, renal-replacement therapy. These patients should be reevaluated in a few days (3-5 days) to assess their response to therapy [15-17]. If they are not responding with continued mechanical ventilation, or have development or persistence of multiorgan system failure, then the patient and/or their family should be approached regarding limiting aggressive therapy and considering palliative care. While aggressive therapy is appropriate in patients with lung cancer, it appears that performing advanced cardiac life support on those with critical illness is futile. Some studies have shown that it is unusual for those patients to survive their hospitalization $[2,4]$. This observation may be conveyed to patients and their family members to avoid subjecting the patients to this measure if their condition deteriorates so as to require it.

'The ICU outc ome of lung cancer patients ... is more dependent on the severity of the acute illness and number of organ failures than the characteristics of the underlying malignancy.'

An important aspect in the care of lung cancer patients is a discussion of the extent of treatment that the patient wishes for and the code status early in the course of illness. It has been our experience, and documented in the literature, that most lung cancer patients admitted to the ICU did not have a discussion with their oncologist regarding the extent of treatment that they wish to have, and did not address their code status. This creates an extra burden and, sometimes, confusion regarding the patient's wishes. C ritically ill lung cancer patients may not be able to make their own decisions and their family may not know the patients' wishes. Thoughtful decision making regarding the endof-life issues is difficult under these stressful situations. In a study by Reichner et al., only $26 \%$ of lung cancer patients admitted to the ICU had 
do-not-resuscitate (DNR) orders, and $64 \%$ of patients with stage IV non-small-cell lung cancer were full code [4]. Of those patients who were full code on admission to the ICU, there was no record of code discussion documented in any of the available outpatient records. The code status was subsequently changed to DN R in $49 \%$ of the patients at a mean of 7 days after admission to the ICU. The pulmonary- and critical-care physician was solely responsible for the change in the code status in $65 \%$ of the cases [4]. Another study by Lamont et al. showed that physicians provide a frank estimate of prognosis in only a third of cases; in the rest of the cases, they provide no estimate or consciously overestimate or underestimate survival time [18]. It may be understandable for the oncologist not to discuss end-of-life issues as they are starting treatment for these patients; however, they are the best-positioned physicians to have a frank discussion with their patients and their families in the outpatient setting after they had a good rapport with them and had time to assess their health and response to therapy. An important resource to physicians caring for lung cancer patients is consultation with the palliative-care service that works with the patient, family and physicians when they are going through treatment and making end-of-life decisions. Recent observations have shown that such services are underutilized, especially in critically ill patients. Furthermore, making use of the palliative care services has been associated with lowered cost for care of cancer patients [19]. The recent American College of $C$ hest Physicians guidelines for the diagnosis and management of lung cancer recommend that palliative care, including a consultation with a palliative-care team, be integrated into the treatment of patients with advanced lung cancer, even those pursuing curative or life-prolonging therapies [20].

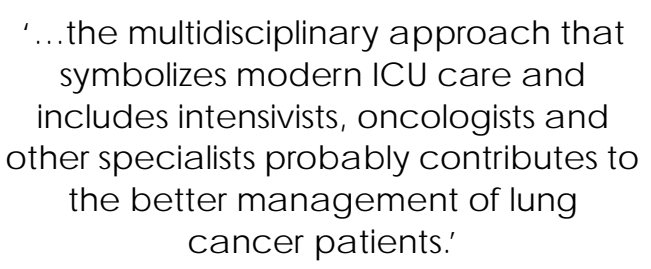

Increasingly, lung cancer patients are admitted to the ICU for conditions related to their malignancy, side effects of treatment or comorbid illnesses. The outcome of these patients has been progressively improving, and is approximating that of the general critically ill population. There are no absolute predictors of outcome of these patients, and full ICU care should be offered to all lung cancer patients who wish to have it, or to those whose wishes are not known, except those who clearly have an advanced refractory disease. It is reasonable to reassess these patients after 3-5 days of their care in the ICU to decide on further management. Every effort should be made to discuss the patient's end-of-life wishes and code status with their oncologist in the outpatient setting.

Financial \& competing interests disclosure The authors have no relevant affiliations or financial involve ment with any organization or entity with a financial interest in or financial conflict with the subject matter or materials discussed in the manuscript. This includes employment, consultancies, honoraria, stock ownership or options, expert testimony, grants or patents received or pending or royalties.

No writing assistance was utilized in the production of this manuscript.

\section{Bibliography}

1. Jemal A, Siegel R, Ward E et al.: Cancer statistics, 2006. CA Cancer J. Clin. 56, 106-130 (2006).

2. Adam AK, Soubani AO : O utcome and prognostic factors of lung cancer patients admitted to the medical intensive care unit. Eur. Respir. J. 31, 47-53 (2008).

3. Soares M, D armon M, Salluh JI et al.: Prognosis of lung cancer patients with life-threatening complications. Chest 131, 840-846 (2007).

4. Reichner $C A$, Thompson JA, O 'Brien $S$, Kuru T, Anderson ED : O utcome and code status of lung cancer patients admitted to the medical ICU. Chest 130, 719-723 (2006).
5. Sharma G, Freeman J, Zhang D, Goodwin JS: Trends in end-of-life ICU use among older adults with advanced lung cancer. Chest 133, 72-78 (2008).

6. Griffin JP, N elson JE, Koch KA et al.: End-of-life care in patients with lung cancer. Chest 123, S312-S331 (2003).

7. Kress JP, Christenson J, Pohlman AS, Linkin D R, H all JB: O utcomes of critically ill cancer patients in a university hospital setting. Am. J. Respir. Crit. Care M ed. 160, 1957-1961 (1999).

8. Jennens RR, Rosenthal M A, M itchell P, Presneill JJ: O utcome of patients admitted to the intensive care unit with newly diagnosed small cell lung cancer. Lung Cancer 38, 291-296 (2002).
9. Lin $Y C$, Tsai $Y H, H$ uang $C C$ et al.: $O$ utcome of lung cancer patients with acute respiratory failure requiring mechanical ventilation. Respir. M ed. 98, 43-51 (2004).

10. Azoulay $E$, Alberti $C$, Bornstain $C$ et al.: Improved survival in cancer patients requiring mechanical ventilatory support: impact of noninvasive mechanical ventilatory support. Crit. Care M ed. 29, 519-525 (2001).

11. Cuomo A, D elmastro $M$, C eriana $P$ et al.: $\mathrm{N}$ oninvasive mechanical ventilation as a palliative treatment of acute respiratory failure in patients with end-stage solid cancer. Palliat. M ed. 18, 602-610 (2004). 
12. Christodoulou C, Rizos M, Galani E, Rellos K, Skarlos DV, M ichalopoulos A: Performance status (PS): a simple predictor of short-term outcome of cancer patients with solid tumors admitted to the intensive care unit (ICU). Anticancer Res. 27, 2945-2948 (2007).

13. Boussat S, El'rini T, D ubiez A, D epierre A, Barale F, Capellier G: Predictive factors of death in primary lung cancer patients on admission to the intensive care unit. Intensive Care M ed. 26, 1811-1816 (2000).

14. Ewer M S, Ali M K, Atta M S, M orice RC Balakrishnan PV: $O$ utcome of lung cancer patients requiring mechanical ventilation for pulmonary failure. JAM A 256, 3364-3366 (1986).

15. Guiguet $\mathrm{M}, \mathrm{B}$ lot $\mathrm{F}$, Escudier $\mathrm{B}$, Antoun $\mathrm{S}$, Leclercq B, N itenberg G : Severity-of-illness scores for neutropenic cancer patients in an intensive care unit: which is the best predictor? D o multiple assessment times improve the predictive value? Crit. Care M ed. 26, 488-493 (1998).
16. Rubenfeld GD, Crawford SW: W ithdrawing life support from mechanically ventilated recipients of bone marrow transplants: a case for evidencebased guidelines. Ann. Intern. M ed. 125, 625-633 (1996).

17. Larche J, Azoulay E, Fieux F et al.: Improved survival of critically ill cancer patients with septic shock. Intensive Care M ed. 29, 1688-1695 (2003).

18. Lamont EB, Christakis N A: Prognostic disclosure to patients with cancer near the end of life. Ann. Intern. M ed. 134, 1096-1105 (2001).

19. Fadul N, Elsayem A, Palmer JL, Zhang T, Braiteh F, Bruera E: Predictors of access to palliative care services among patients who died at a $\mathrm{C}$ omprehensive $\mathrm{C}$ ancer $\mathrm{C}$ enter. J. Palliat. M ed. 10, 1146-1152 (2007).

20. Griffin JP, Koch KA, N elson JE, Cooley M E: Palliative care consultation, quality-of-life measurements, and bereavement for end-of-life care in patients with lung cancer: ACCP evidence-based clinical practice guidelines (2nd edition). Chest 132, S404-S422 (2007).
Affiliations

- Ayman 0 Soubani, MD, Associate Professor of $M$ edicine Karmanos $C$ ancer $C$ enter and Wayne State U niversity School of $\mathrm{M}$ edicine, Division of Pulmonary, Allergy, Critical Care and Sleep M edicine, 3990 John R- 3 H udson, D etroit, M I 48201, U SA Tel.: +1 3137458471 Fax: +1 3139930562 asoubani@med.wayne.edu

\section{- Abdulgadir K Adam, M D} Karmanos $\mathrm{Cancer}$ Center and Wayne State University School of M edicine Division of Pulmonary, Allergy, Critical Care and Sleep M edicine, 3990 John R- 3 H udson, D etroit, M I 48201, U SA 